\title{
WIND-INDUCED RESPONSE OF LARGE-SPAN STRUCTURES BASED ON POD-PSEUDO-EXCITATION METHOD
}

\author{
Zhongwei Zhao ${ }^{2}$, Zhihua Chen ${ }^{1,2,3}$, Xiaodun Wang ${ }^{2,}{ }^{*}, \mathrm{HaoXu}{ }^{2}$ and Hongbo Liu ${ }^{2}$ \\ ${ }^{1}$ State Key Laboratory of Hydraulic Engineering Simulation and Safety, \\ Tianjin University, Tianjin 300072, China \\ ${ }^{2}$ School of Civil Engineering, Tianjin University, Tianjin 300072, China; \\ ${ }^{3}$ Key Laboratory of Coast Civil Structure and Safety of Ministry of Education, Tianjin University, \\ Tianjin 300072, China; \\ *(Corresponding author: E-mail:maodun2004@126.com)
}

Received: 12 January 2014; Revised: 3 June 2015; Accepted: 18 June 2015

\begin{abstract}
This paper proposes a new method to analyze wind-induced response in frequency domain for long-span spatial structures. This method is based on proper orthogonal decomposition (POD) and pseudo-excitation methods, which could significantly reduce computational cost. Based on the POD-pseudo-excitation method and the wind pressure coefficient obtained from a wind tunnel test, ANSYS general purpose software package was developed for the analysis of wind-induced response of YUJIAPU Railway Station Building, which is designed in the form of a conch-shaped single-layer lattice shell. The wind vibration coefficient in wind direction of $0^{\circ}$ was obtained. Results obtained by frequency- and time-domain methods were compared. The results were consistent with each other, establishing the foundation for further application of these methods in wind-induced analysis. The relationship between wind-induced response and the frequency of wind load was also determined. This paper provides effective guidance on equivalent wind load.
\end{abstract}

Keywords: POD-pseudo-excitation method, wind vibration coefficient, wind-induced response, harmonic response analysis, conch-shaped single-layer lattice shell

DOI: $10.18057 /$ IJASC.2016.12.1.1

\section{INTRODUCTION}

Long-span roofs are widely used in large-scale buildings such as gymnasiums, exhibition centers, airports, and railway stations because such roofs provide a large space without inner columns and offer architects and designers an alternative to regular rectangular building forms. These kinds of roof structures comprise light materials with small damping ratios and low natural frequencies. They are sensitive to wind loading actions because of their light weight and small structural rigidity [1]. Wind-induced response analysis is an important and interesting issue for theoretical study and structural design.

Extensive studies have been conducted and remarkable developments have been achieved on wind resistance of long-span structures. The approach on the effects of wind loads on structures mainly includes time and frequency domain methods. Time-history curves must be obtained using a specified method. The time domain method is adopted because it is easy to understand and use. However, the method requires more computational resources, i.e., high cost and low efficiency. The advantage of the frequency domain method over time the domain method is its high calculation efficiency. The core problem in its application is that a number of modes of vibration rather than only the first mode must be considered in the analysis of wind-induced response particularly for long-span structures. Furthermore, the cross correlation terms between normal modes in a structural response [2], which are often ignored in previous studies, may have to be retained because long-span structures possess closely spaced natural frequencies and normal modes. Simultaneously, 
the contribution of high-order modes of vibration to the response of structures is not always less than low-order modes.

YUJIAPU Railway Station building in Tianjin, China has an area of $86200 \mathrm{~m}^{2}$. The three-story building with complex roof shapes has two basement floors. The station hall, including waiting and entrance halls, device rooms, and an office, is on the first floor. The platform with three island platforms and six arrival-departure tracks are on the second floor. The building has a conch-shaped single-layer lattice lighting roof. The main members of the entire structure are 72 curved steel box girders that intersect one another, as shown in Figure 4. Figure 5 shows the construction process of the station building that is $142 \mathrm{~m}$ in length and $80 \mathrm{~m}$ in width, and with vector height of $24 \mathrm{~m}$. A ring beam was set on top and bottom of the shell to connect and constrain the steel box girders.

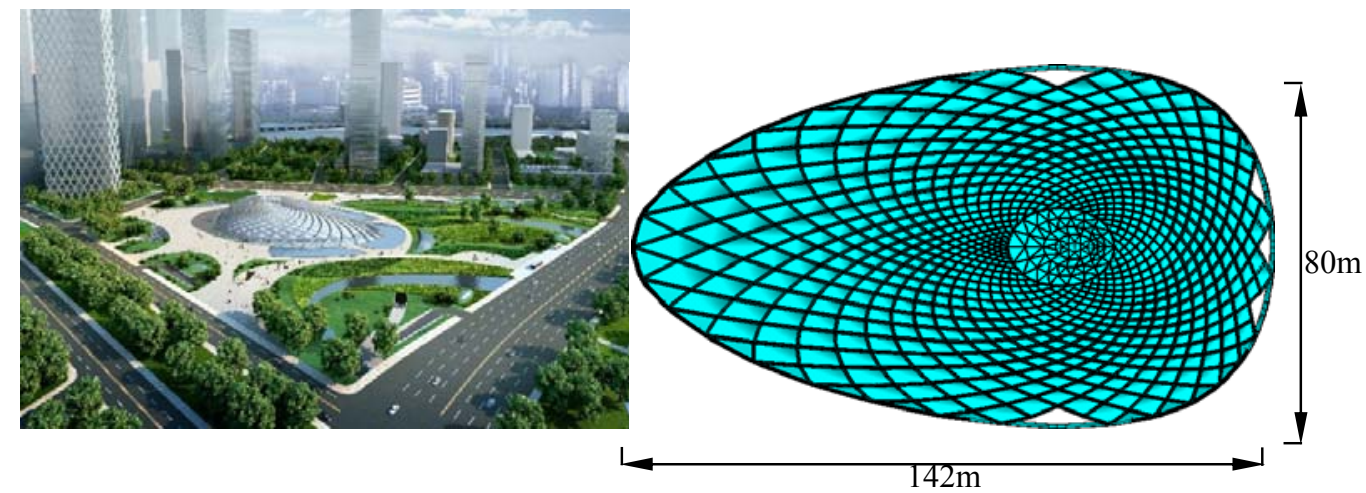

a)

b)

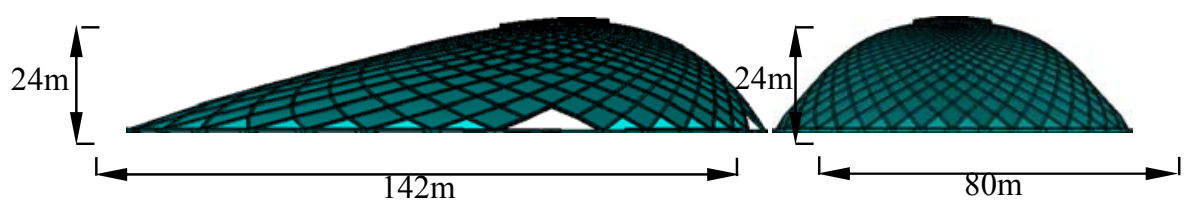

c)

d)

Figure 1. Details of the Structure. a) Aerial View of YUJIAPU Railway Station Building b) Plan view, c) Lateral View, d) Front View

Studies have been conducted on wind-excited multi-story shear buildings and towers [3-4]. Zhou et al. [5] focused on the wind-induced vibration response of pre-stressed double-layer spherical latticed shell structures by adopting time-domain analysis method. Chen et al. [6] analyzed the wind-induced responses of long-span bridges by considering coupled multimode effect. Lin [7] proposed a pseudo-excitation method to address the dynamic response of structures subject to random seismic excitation. In this method, determining the random response of a structure is converted into finding the response of the structure under a series of harmonic loads, i.e., the so-called pseudo-excitations. $\mathrm{Xu}$ et al. [8] decomposed the excitation spectral matrix by LDLT method and analyzed the wind-induced response of high-rise structures. However, these methods are unsuitable for analysis of long-span spatial structures because of computational cost. The background response was significantly higher than the mean response and the resonant response; low-order eigenmodes and vibration modes provided the most important contributions to the wind-induced response [9]. Yang et al. [10] developed a program to analyze the wind-induced vibrations as a result of a combination of wind vibration modes, but did not consider the spatial distribution of wind load. The multi-mode coupling effect was considered in computing the resonant buffeting response and equivalent static wind loads. Zhou and $\mathrm{Gu}$ [11] used the modified 
SRSS method, but the computational cost was high. Zhou [12] adopted the neural network method to predict the mean wind pressure coefficients and the power spectra of the fluctuating wind pressures on a real large-span roof based on the limited data from the wind tunnel test. However, the data from the wind tunnel test was extremely limited, affecting the accuracy of the neural network method. Yang [13] validated that the background responses were much larger than the resonant responses.

No method was highly efficient and accurate in wind induced analysis and can be conducted in the frequency domain for spatial structures. This paper provides a new method suitable for spatial structures, which can reduce computational cost significantly.

\section{ANALYSIS THEORY}

\subsection{Pseudo-excitation Method}

The pseudo-excitation method proposed by Lin [7-9] was adopted to address the dynamic response of structures subject to random wind load. This method involves the cross-correlation terms between normal modes automatically and considers non-complete correlation among multi-support excitation. The method is equal to complete quadratic combination (CQC) in the mathematical sense, and the computational cost is much less than that of CQC, and even less than the square root of the sum of the squares (SRSS), which cannot consider the cross correlation terms between normal modes. The pseudo-excitation method proved to be an accurate and quick computing method.

Wind load acting on the building surface includes the mean wind loads $F$ and the fluctuating wind loads $F(\mathrm{t})$. The equation of motion of a linear and elastic structure under the action of fluctuating wind forces $F(\mathrm{t})$ in terms of finite element technique can be expressed as

$$
M \ddot{Y}(t)+C \dot{Y}(t)+K Y(t)=R F(t)
$$

where $M, C$, and $K$ are the $N \times N$ system mass, damping, and stiffness matrices, respectively, $Y(t)$ is the displacement vector, $F(t)$ is the wind force vector of $m$ dimension $(m \times N), R$ is the $N \times m$ matrix consisting of 0 or 1 , which expands the $m$-dimensional loading vector into the $N$-dimensional loading vector, and a dot represents the first-order derivative with respect to time $t$.

The Fourier transformation of Eq. 1 provides the transfer function between the loading and displacement response.

$$
H(i w)=\left[-w^{2} M+i w C+K\right]^{-1}
$$

where the superscript -1 indicates the matrix inversion and $i$ is the imaginary unit.

Assuming that the power spectrum of wind load at a certain point is $S_{f}(w)$, we substitute this random excitation by pseudo harmonic excitation as expressed by Eq. 3 .

$$
x(t)=\sqrt{S_{f}(w)} e^{i w t}
$$

The steady-state response of structures with certain natural vibration characteristics can be derived as 
$\{y\}=\sum_{j=1}^{q} \gamma_{j} H_{j}\left\{\phi_{j}\right\} \sqrt{S_{f}(w)} e^{i w t}$

Furthermore, we obtain the power spectral density of response as

$\left[S_{y y}(w)\right]=\{y\}^{*}\{y\}^{T}=\sum_{i=1}^{q} \sum_{j=1}^{q} \gamma_{i} \gamma_{j} H_{i}^{*} H_{j}\left\{\phi_{i}\right\}\left\{\phi_{j}\right\}^{T} S_{f}(w)$

The pseudo-excitation method is validated to be equal to the CQC method.

\subsection{Model of Wind Load Spectrum}

This study uses the Kaimal wind speed spectrum, which can consider the effect of altitude change as fluctuating wind speed spectrum and is expressed as

$$
\left\{\begin{array}{l}
S_{v}(w)=\frac{200 u_{*}{ }^{2} \xi}{w(1+50 \xi)^{5 / 3}} \\
u_{*}=k \bar{v}_{10} / \ln \left(10 / z_{0}\right), \xi=z w / \overline{v_{z}}
\end{array}\right.
$$

where $k, \mathrm{z}_{0}, \bar{v}_{10}, u^{*}, \mathrm{z}$, and $\alpha$ are Kaimal constant, the rough length of ground, mean wind speed of $10 \mathrm{~m}$ altitude, wind friction speed, and height of the wind speed, respectively; and $w$ is the frequency of the wind speed.

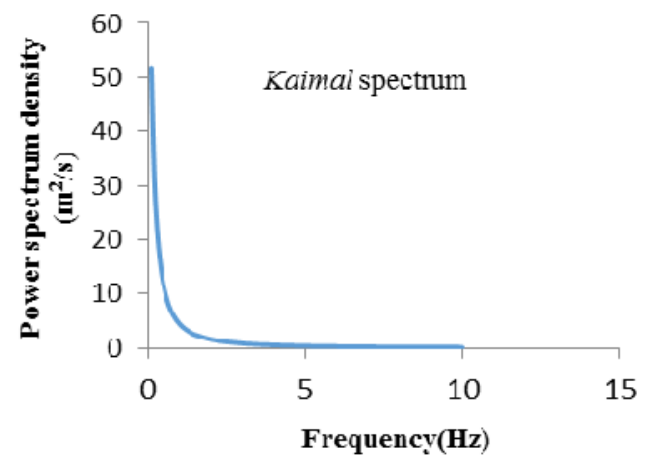

Figure 2. Wind Speed Spectrum at Node 2

The wind speed spectrum shows that the fluctuating wind contains minimal high-frequency components. We assume that the wind-induced response caused by high-frequency components is so small that we can neglect the response when the flexible long-span structures are subjected to high-frequency components. This study examines the relationship between wind-induced response and frequency of wind load to validate whether we can neglect this part of the response.

The wind speed and direction at each point is not synchronous and are even completely independent. The spatial correlation of gusty wind can be divided into lateral and vertical correlations, even before and after correlation. The tests indicate a negative correlation with decay with exponential rates and distance between two nodes. The cross power spectrum can consider this kind of correlation in actual engineering, and the cross power spectrum can be expressed as Eq. 7. 


$$
S_{v v}(w)=\left[\begin{array}{cccc}
S_{v_{1} v_{1}} & S_{v_{1} v_{2}} & \cdots & S_{v_{1} v_{n}} \\
S_{v_{2} v_{1}} & S_{v_{2} v_{2}} & \cdots & S_{v_{2} v_{n}} \\
\vdots & \vdots & \ddots & \vdots \\
S_{v_{n} v_{1}} & S_{v_{n} v_{2}} & \cdots & S_{v_{n} v_{n}}
\end{array}\right]
$$

where

$$
\begin{aligned}
& S_{v_{i} v_{j}}\left(x_{1}, x_{2}, y_{1}, y_{2}, z_{1}, z_{2}, w\right)=\rho_{i j}\left(x_{1}, x_{2}, y_{1}, y_{2}, z_{1}, z_{2}, w\right) \cdot \sqrt{S_{v_{i}}\left(x_{1}, y_{1}, z_{1}, w\right) S_{v_{j}}\left(x_{1}, y_{1}, z_{1}, w\right)} \\
& \rho_{i j}\left(x_{1}, x_{2}, y_{1}, y_{2}, w\right)=\exp \left(\frac{-2 n\left[C_{x}^{2}\left(x_{i}-x_{j}\right)^{2}+C_{y}^{2}\left(y_{i}-y_{j}\right)^{2}+C_{z}^{2}\left(z_{i}-z_{j}\right)^{2}\right]}{\bar{v}_{i}+\bar{v}_{j}}\right)
\end{aligned}
$$

where $S_{v_{i} v_{j}}\left(x_{1}, x_{2}, y_{1}, y_{2}, w\right)$ is the cross power spectrum of nodes $i$ and $j ; \rho_{i j}\left(x_{1}, x_{2}, y_{1}, y_{2}, w\right)$ is coherence function; $C_{\mathrm{x}}, C_{\mathrm{y}}$, and $C_{\mathrm{z}}$ are constants equal to 16,8 , and 10, respectively; $\overline{v_{i}}$ and $\overline{v_{j}}$ are mean wind speed at nodes $i$ and $j$.

\subsection{Expression of Wind Load}

Natural wind is classified according to frequency into mean and fluctuating winds. The effect of mean wind indicates that long period components are similar to static load, and the dynamic effect should be considered for fluctuating wind. Wind speed and the equivalent nodal load expressed by wind speed are as follows:

$$
\begin{aligned}
& V(t)=\bar{v}+v(t) \\
& F_{i}(z, t) \\
& =\frac{1}{2} \mu_{s i} A_{i} \rho V^{2}(t) \\
& =\frac{1}{2} \mu_{s i} A_{i} \rho \bar{V}^{2}+\mu_{s i} A_{i} \rho \bar{V} v(z, t)+\frac{1}{2} \mu_{s i} A_{i} \rho v(z, t)^{2}
\end{aligned}
$$

where $F_{\mathrm{i}}(z, t), \mu_{s i}, A_{i}$ are nodal load, shape coefficient, and tributary area of node $i$, respectively. $v(z$, $t$ ) is the wind speed at time $t$, and $\bar{v}$ is the mean wind speed at $z$ altitude.

The first term in Eq. 11 is the equivalent nodal load caused by mean wind. As the fluctuating wind speed is extremely small compared to the mean wind speed, the third term can be ignored. Then, the equivalent nodal load, which is obtained based on quasi-steady assumption of node $i$ can be expressed as follows [10]

$$
f_{i}(t)=\mu_{s i} A_{i} \rho \bar{V}_{i} v_{i}(t)=B_{i} v_{i}(t)
$$

where

$$
B_{i}=\mu_{s i} A_{i} \rho \bar{V}_{i}
$$


The direction of $f_{i}(t)$ is along the normal vector of the surface of structure. Then, $f_{i}(t)$ is decomposed along the global coordinate through which we obtain an equivalent nodal load expressed in three orthogonal directions.

$$
\left\{\begin{array}{l}
f_{i x}(t) \\
f_{i y}(t) \\
f_{i z}(t)
\end{array}\right\}=\left\{\begin{array}{l}
B_{i} v_{i x}(t) \\
B_{i} v_{i y}(t) \\
B_{i} v_{i z}(t)
\end{array}\right\}=\left\{B_{i}\right\} *\left\{\begin{array}{l}
v_{i x}(t) \\
v_{i y}(t) \\
v_{i z}(t)
\end{array}\right\}
$$

where $v_{i x}(t)=\sqrt{v_{i}(t)^{2} \cos \alpha}, \quad v_{i y}(t)=\sqrt{v_{i}(t)^{2} \cos \beta}, v_{i z}(t)=\sqrt{v_{i}(t)^{2} \cos \gamma}, \alpha, \beta$, and $\gamma$ are angles between the normal vector of surface and $x$-axis, $y$-axis, and $z$-axis of global coordinate, '.$^{*}$ ' is matrix operator of MATLAB, indicating the multiplication of corresponding elements of two matrices with the same number of rows and columns.

The integration of Eq. 14 on all nodes can be written in matrix form as follows:

$\{(t)\}=[B v(t)\}$

where $\{f(t)\}=\left\{f_{1 x}(t), f_{1 y}(t), f_{l z}(t) \cdots, f_{n x}(t), f_{n y}(t), f_{n z}(t)\right\}$ is a $3 n \times 1$ matrix; $[B]=\operatorname{diag}(\{B\})$ is a $3 n$ $\times 3 n$ matrix; and $\{B\}=\operatorname{diag}\left(\left\{B_{i}\right\}\right),\{v(t)\}=\left\{v_{l x}(t), v_{l y}(t), v_{l z}(t) \cdots, v_{n x}(t), v_{n y}(t), v_{n z}(t)\right\}$ is a $3 n \times 1$ matrix.

\subsection{Proper Decomposition Technique}

Proper decomposition technique is an effective method to analyze random field. This method is used to obtain the intrinsic mode, which represents the spatial distribution pattern of fluctuating wind [14].

The main objective of the proper decomposition technique is to find deterministic function $\Phi(x, y)$, which is best correlated with all the elements of the ensemble of a random field. Given a random pressure $p(x, y, t)$, the maximum projection of $p(x, y, t)$ on the function $\Phi(x, y)$ is sought by considering an inner product

$\frac{\int p(x, y, z) \phi(x, y) d x d y}{\int \phi(x, y)^{2} d x d y}=\max$

The maximization of the function projection expressed by Eq. 17 is performed in the mean-square sense as follows:

$\frac{\left.\left\langle\iint p(x, y, z) \phi(x, y) d x d y\right)\left(\int p\left(x^{\prime}, y^{\prime}, z^{\prime}\right) \phi\left(x^{\prime}, y^{\prime}\right) d x^{\prime} d y^{\prime}\right)\right\rangle}{\int \phi^{2}(x, y) d x d y}$

$=\lambda>0$

This equation leads to an eigenvalue problem [15],

$\int R_{p}\left(x, y, x^{\prime}, y^{\prime}\right) \phi\left(x^{\prime}, y^{\prime}\right) d x^{\prime} d y^{\prime}=\lambda \phi(x, y)$ 
where $R_{\mathrm{p}}\left(x, y, x^{\prime}, y^{\prime}\right)$ is the space correlation of the pressure $p(x, y, t)$. The integration is performed numerically if a discrete pressure field is specified. When the pressure is given at uniformly spaced locations and a rectangular integration quadrature is employed, Eq. 18 is replaced with a matrix equation.

$\left[S_{v v}(w)\right]\{\phi\}=\lambda\{\phi\}$

where $\left[S_{v v}(w)\right]$ is the Davenport's wind speed spectrum correlation matrix, and $\{\phi\}$ and $\lambda$ are a vector and a value to be determined. The eigenvalue indicates the degree of similarity between the eigenvector and spatial correlative random wind field.

\section{POD-PSEUDO-EXCITATION METHOD}

$\mathrm{Xu}$ et al. [10] decomposed the excitation spectral matrix by LDLT method and analyzed the wind-induced response of high-rise structures. However, this method requires considerable computational cost to analyze long-span spatial structures because these kinds of structures often possess thousands of nodes. If the LDLT method is used to decompose this excitation spectral matrix as shown in Eq. 20

$S_{v v}(w)=[L]^{*}[D][L]^{T}$

then the scale of $[L]$ becomes huge. Every column of $[L]$ is considered as a sub power spectrum of the excitation spectral matrix, and $N$ times for every $w$ should be calculated. $N$ is the number of nodes in the structure. The computation is highly expensive if the LDLT method is adopted. The number of nodes in long-span spatial structures can reach several thousands, implying that they require thousands times of operations for every $w$ and cannot take advantage of the pseudo-excitation method.

This paper combines the proper decomposition technique with the pseudo-excitation method, which is called the POD-pseudo-excitation method, to analyze the wind-induced response.

First, the excitation spectral matrix obtained from Eq. 7 is decomposed with proper decomposition technique, as shown in Eq. 21.

$S_{v v}(w)=[P]^{*}[D][P]^{T}$

where $[P]$ is the eigen matrix consisting of eigenvector $S_{v v}(w),[D]$ is a real diagonal matrix consisting of eigenvector, ' $*$ ' is matrix conjugate transformation, and ' $T$ ' 'is matrix transposition.

The nodal force spectrum is obtained using Eqs. 14 and 21.

$$
\begin{aligned}
& S_{f}(w) \\
& =[B][P]^{*}[D][P]^{T}[B]^{T} \\
& =[B][P]^{*}[\sqrt{D}]^{T}([B][P][\sqrt{D}])^{T}
\end{aligned}
$$

We assume that $[F]=[B][P][\sqrt{D}]$. Then, every column of $[F]$ is considered as a group of random excitation. The $k$ th pseudo-harmonic excitation can be expressed as 
$\left\{f_{k}(t)\right\}=\left\{F_{k}\right\} \exp (i \omega t)$

This paper uses large eigenvalues, implying more contributions to structural responses and the corresponding eigenvector in the calculation of wind-induced response of YUJIAPU Railway Station building. The eigenvalue indicates the degree of similarity between the eigenvector and random wind field. Only part of the eigenvalue and the corresponding eigenvectors are used to represent the entire fluctuating wind field. The first 12-order eigenvectors of the wind speed spectrum when $w$ is $0.1 \mathrm{~Hz}$ are shown in Figures $3\left(\lambda_{1}>\lambda_{2}>\lambda_{3}>\cdots>\lambda_{n-1}>\lambda_{n}\right)$. The eigenvectors that correspond to large eigenvalues reflected the holistic distribution characteristics of the wind field, and the eigenvectors reflect the detail with the decrease in eigenvalue.

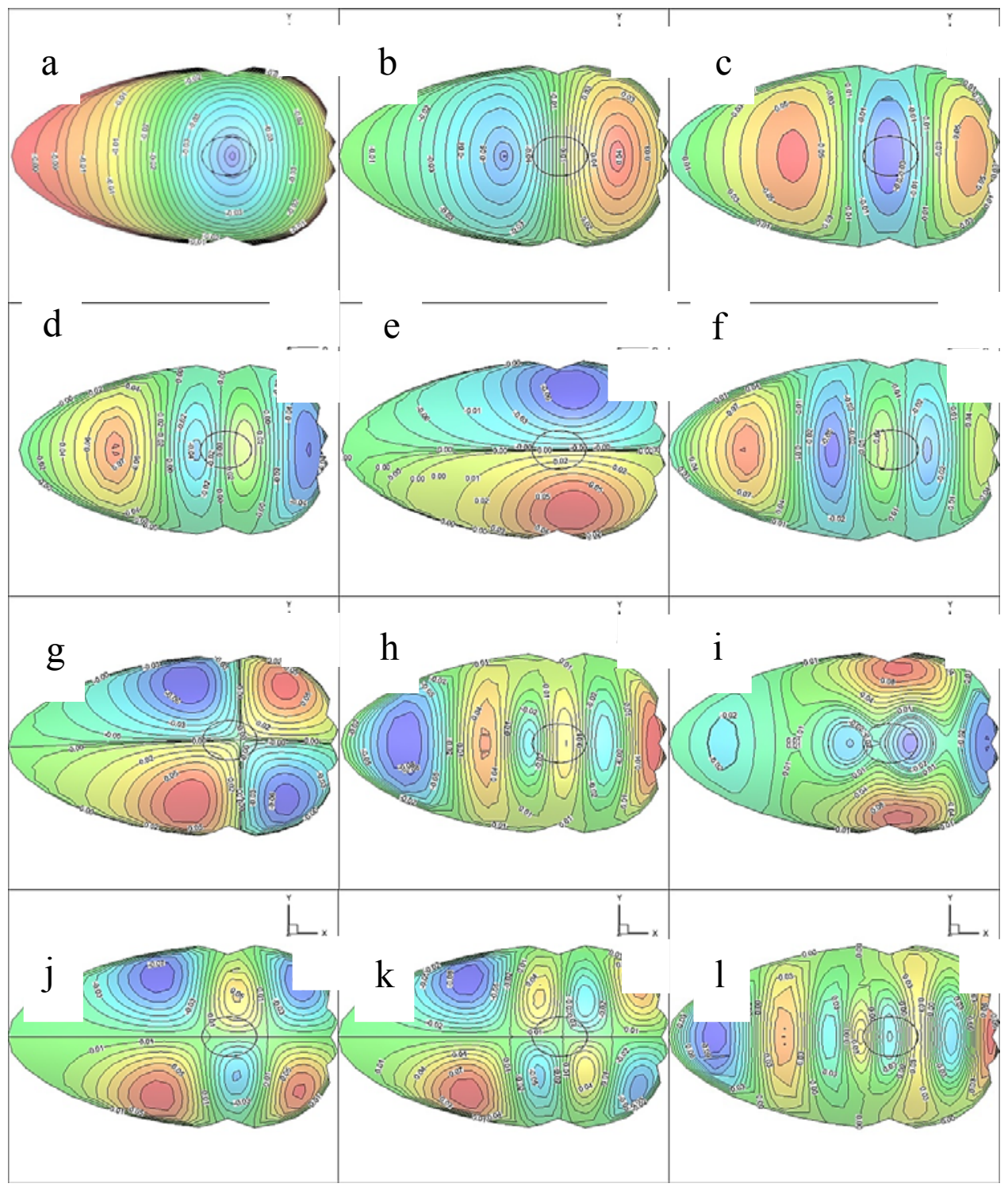

Figure 3. Contour of the First 12-Oorder Eigenvectors of Wind Speed Spectrum $(\mathrm{w}=0.1 \mathrm{~Hz})$. (a) eigenvector 1: eigenvalue $=20168$, (b) eigenvector 2: eigenvalue $=7609$, (c) eigenvector 3 : eigenvalue $=3618$, (d) eigenvector 4: eigenvalue $=2381$, (e) eigenvector 5: eigenvalue $=2283$, (f) eigenvector 6: eigenvalue $=1399,(\mathrm{~g})$ eigenvector 7 : eigenvalue $=1199,(\mathrm{~h})$ eigenvector 8 : eigenvalue $=1011$, (i) eigenvector 9: eigenvalue $=784,(\mathrm{j})$ eigenvector 10 : eigenvalue $=780,(\mathrm{k})$ eigenvector 11: eigenvalue $=666,(1)$ eigenvector 12 : eigenvalue $=552$ 


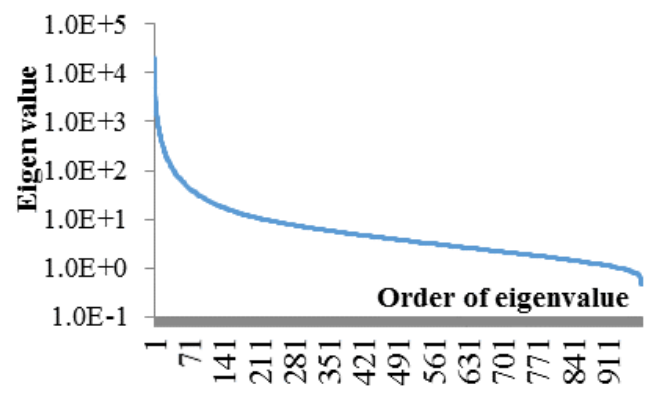

Figure 4. First 12-Order Eigenvalue of Wind Speed Spectrum $(w=0.1 \mathrm{~Hz})$

Figure 4 shows the changing trend in eigenvalue with the order. The eigenvalue decreased rapidly with the order, and thus the first $r$-order eigenvectors and eigenvalues accurately represent the stochastic wind field. Pseudo-harmonic excitation is substituted into the structural vibration differential equation as

$[M]\left\{\ddot{u}_{k}\right\}+[C]\left\{\dot{u}_{k}\right\}+[K]\left\{u_{k}\right\}=\left\{f_{k}(t)\right\}$

Rayleigh damping, $C=\alpha[\mathrm{M}]+\beta[\mathrm{K}]$, is adopted. $\alpha$ and $\beta$ are constants determined by the following equation:

$\alpha=2 * \omega_{1} * \omega_{2} * \xi /\left(\omega_{1}+\omega_{2}\right)$

$\beta=2 * \xi /\left(\omega_{1}+\omega_{2}\right)$

where $\omega_{1}$ and $\omega_{2}$ are circular frequency of the first- and second-order modes, and $\xi$ is damping ratio of 0.02 for steel structures.

For each pseudo-excitation vector, a pseudo-displacement response vector, $\{u \mathrm{k}\}$, can be determined by

$\left\{u_{k}\right\}=\sum_{j=1}^{n} H_{j}(i w)\left\{\phi_{j}\right\}^{T}\left\{f_{k}(t)\right\}$

where $n$ is the number of modes used in calculation. $\left\{u_{\mathrm{k}}\right\}$ can be obtained through Eq. 25 . Then, the response power spectrum is derived by

$\left\{S_{u}(w)\right\}=\sum_{k=1}^{r}\left\{\left|u_{k}\right|\right\}^{2}$

where $r$ is a parameter to be determined, which indicates the number of group of random excitations to be used in wind-induced response analysis.

The wind vibration coefficient is obtained by Eq. 27.

$$
\begin{gathered}
\beta=1+\frac{\mu \sigma}{\bar{U}} \\
\sigma_{u}=\sqrt{\int_{0}^{+\infty} S_{u}(w) d w}
\end{gathered}
$$


where $\beta$ is the wind vibration coefficients of displacement at a certain node, $\mu$ is peak guarantee factor of 3.5, $\bar{U}$ is algebraic average of displacement obtained using time domain analysis, and $\sigma_{u}$ is the mean square root value of displacement response.

With the complexity of the conch-shaped single-layer lattice shell, determining the surface normal direction is an intricate process. This paper obtained the response power spectrum by combining the harmonic response analysis module and secondary development of finite element software package ANSYS. The wind vibration coefficient was obtained using MATLAB.

\section{RESULTS AND COMPARISON}

\subsection{Structural Natural Vibration Characteristics}

The software package ANSYS is used to obtain the natural vibration characteristics through modal analysis. The first six-order modes and natural vibration frequency are shown in Figures 7-8.

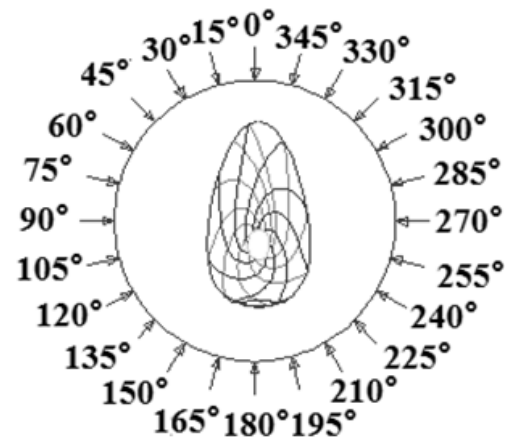

Figure 5. Sketch Map of Wind Direction

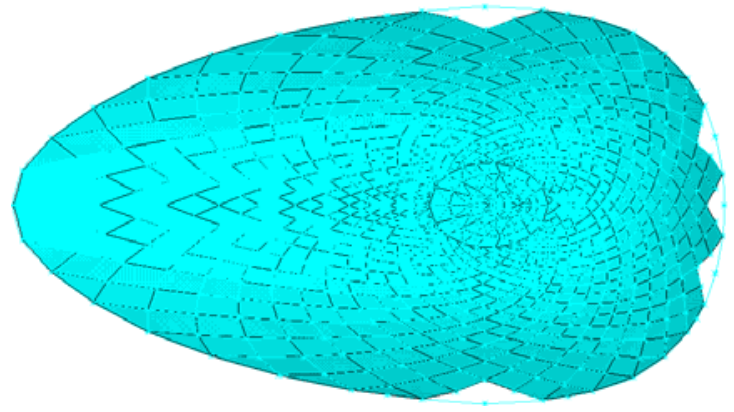

Figure 6. Structure Finite Element Model

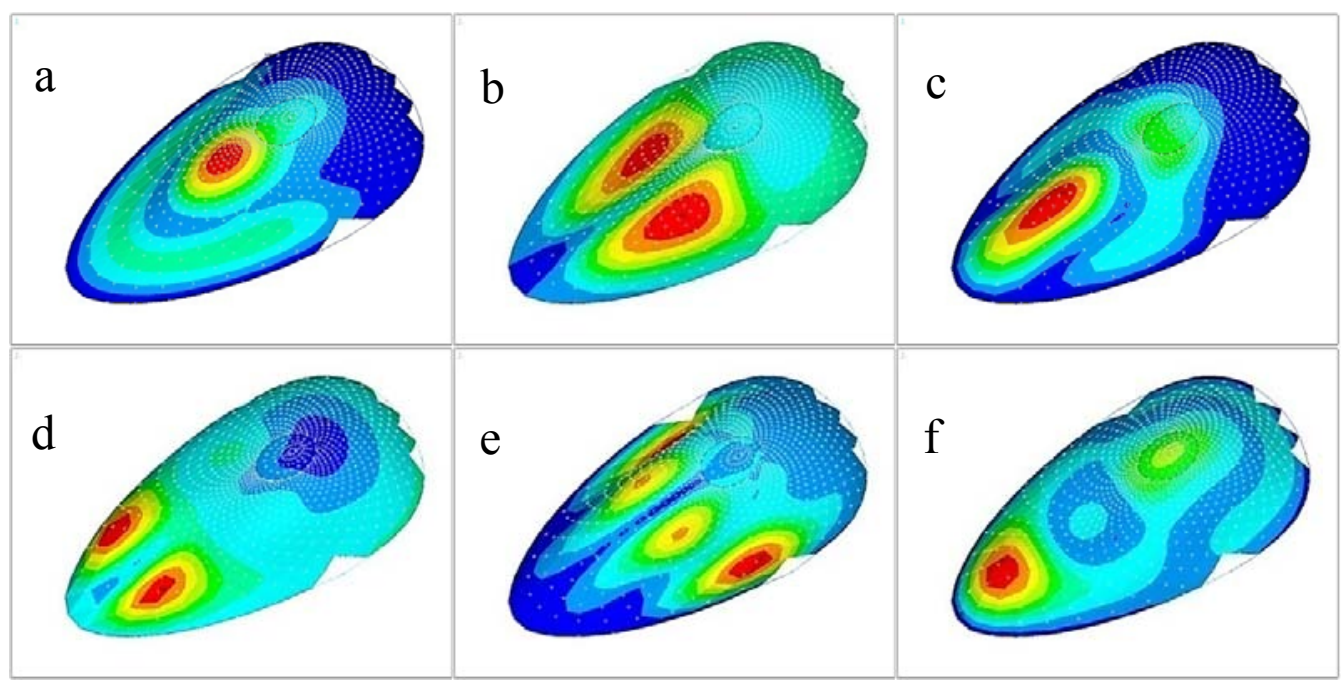

Figures 7. Mode Shapes of the First Six Modes. (a) Mode 1:f1 $=1.562 \mathrm{~Hz}$, (b) Mode 2:f2 = 1.644 Hz, (c) Mode 3:f3 = 2.113 Hz, (d) Mode 4:f4 = 2.166 Hz, (e) Mode 5:f5 = 2.303 Hz, (f) Mode 6:f6 = 2.537 Hz. 


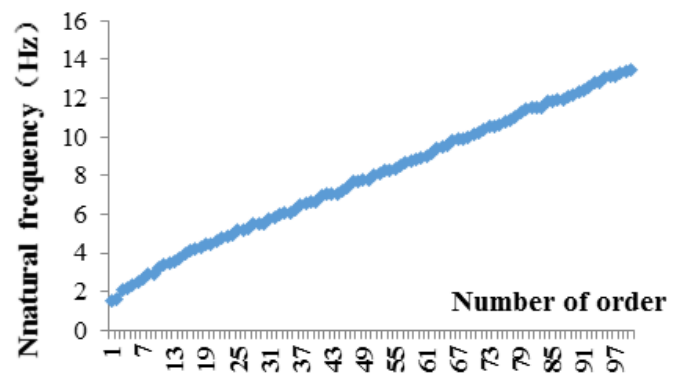

Figure 8. Distribution of the First 100 Natural Frequencies

\subsection{Wind Tunnel Test}

This study conducts a wind-induced response analysis for YUJIAPU Railway Station building in wind direction of $0^{\circ}$. The wind pressure coefficient obtained through a wind tunnel test is shown in Figure 10. The equivalent nodal wind load was obtained by substituting pressure coefficient into Eq. 12.

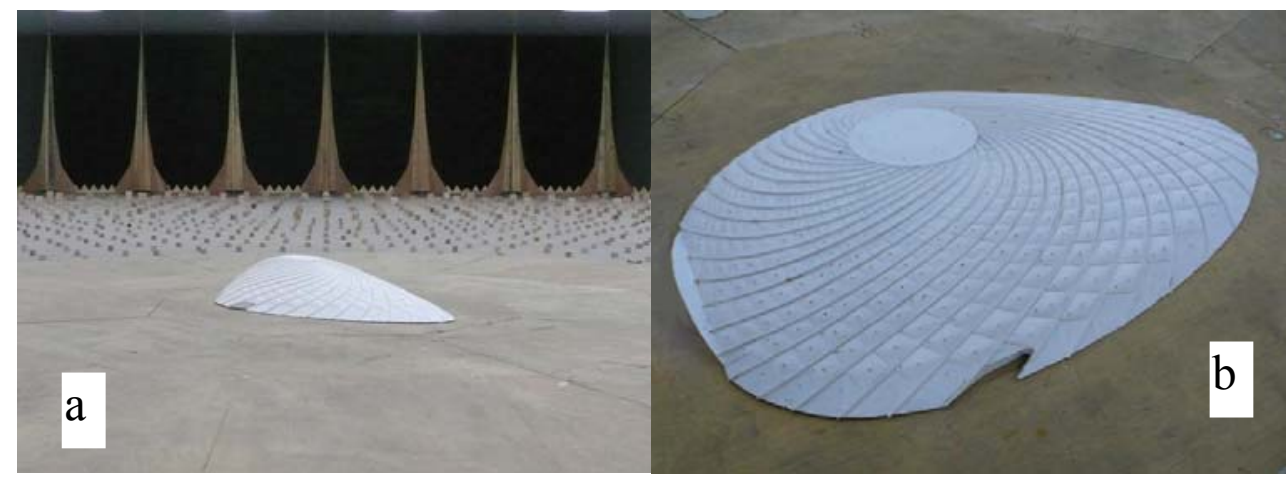

Figures 9. Wind Tunnel Test Model (a) Overview, (b) Close-up view

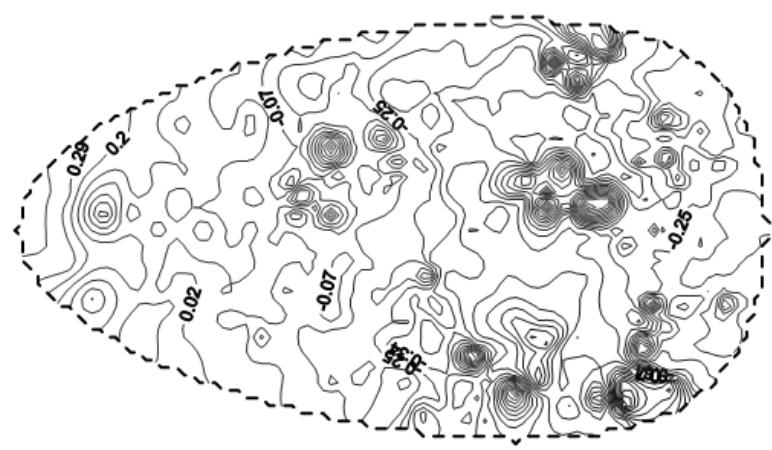

Figure 10. Contour of Pressure Coefficient

\subsection{Change of Wind-induced Response with Number of Eigenvectors}

For comparison, linear filtering method and the same Kaimal spectrum, which were used in POD-pseudo-excitation method generated the wind load time history, which is used in wind-induced response analysis in the time domain. The wind vibration coefficient was obtained with dynamic time history analysis by using ANSYS. Refer to other documents[16-17] for the detailed process of linear filtering method. Figure 11 shows the wind speed time series and power spectrum obtained from the linear filtering method. 


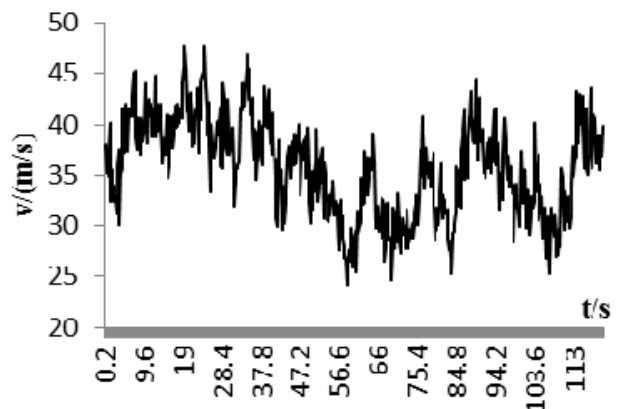

(a)

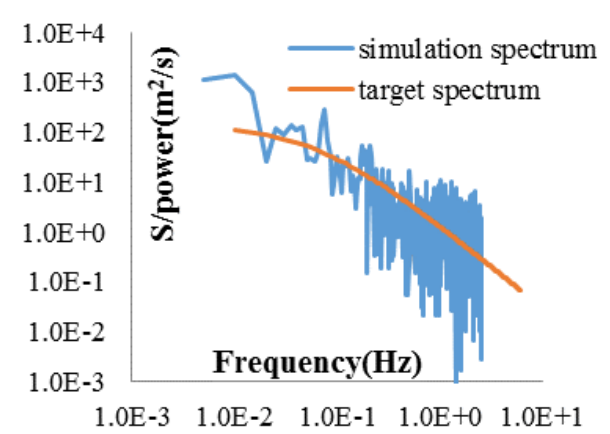

(b)

Figure 11. Simulated Wind Speed of Element 2901 (a) Time History of Wind Speed (b) Comparison of Simulation Spectrum and Target Spectrum

To obtain the minimum $r$ under the condition that satisfies the calculation accuracy, the relationship between wind-induced displacement response and the number of $r$ (i.e., the number of pseudo-excitation groups used in the analysis) were examined, as shown in Figure 13. The mean square deviation of displacement did not change when $r$ reached a certain degree. Thus, the response induced by the random excitation represented by a higher order of eigenvectors can be ignored, and the accuracy of results is not affected.

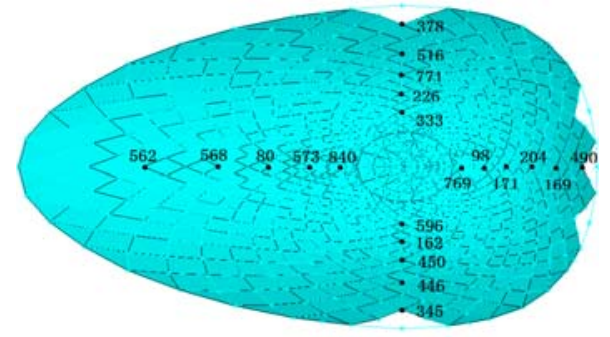

Figure 12. Number of Nodes Located at Typical Position
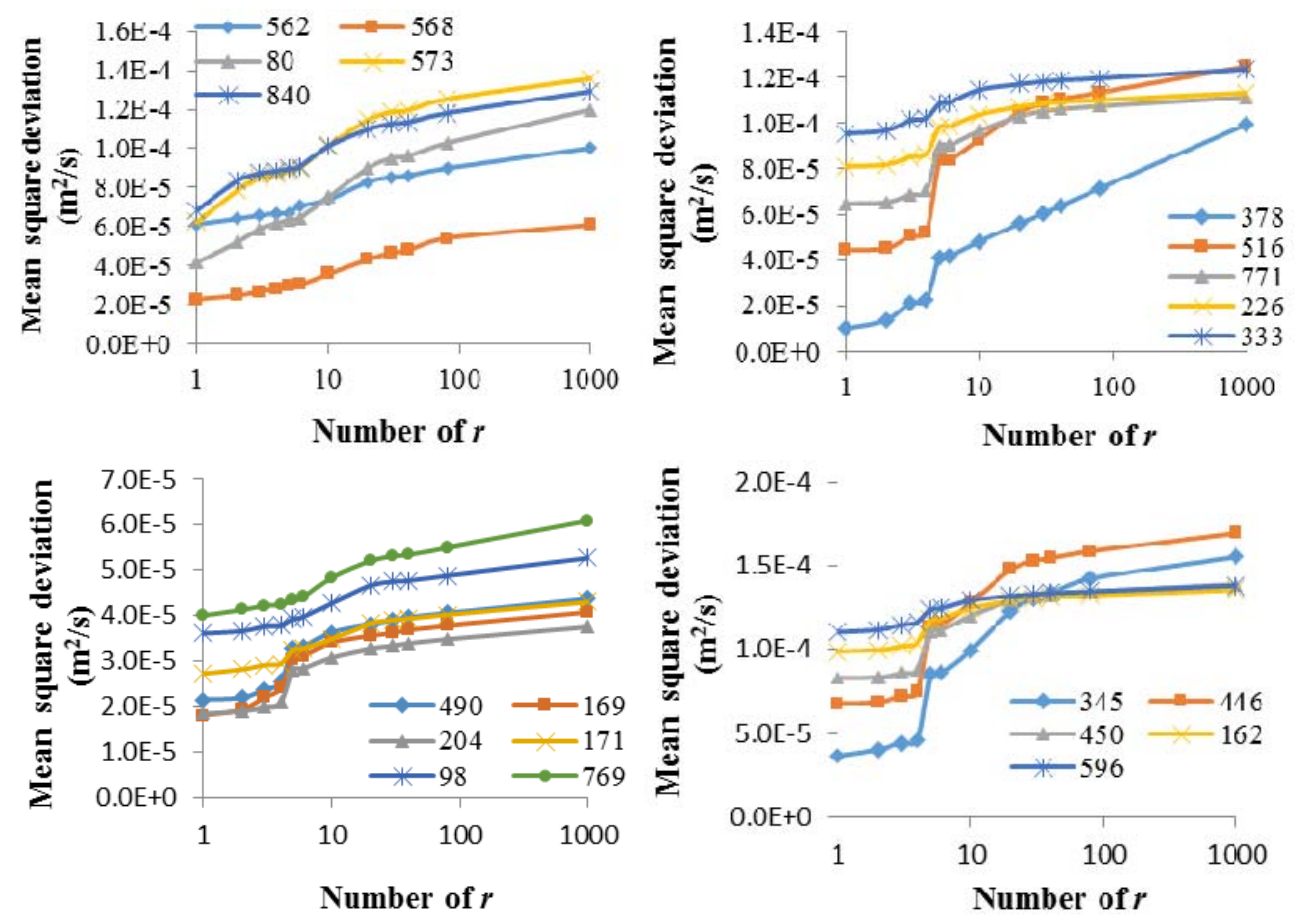

Figure 13. Relationship between Response and Number of $r$ 
For the purpose of quantitative research, we assume that $\lambda_{\text {sum }}=\sum_{i=1}^{n} \lambda_{n}, n$ is the number of structural nodes for general situation, $\rho_{i}=\lambda_{i} / \lambda_{\text {sum }}$ which indicates the weight of the $i$ th group of random excitation represented by the $i$ th order eigenvector. Figure 14 shows that the weight of the first 20 groups of random excitation can reach $80 \%$, and the weight of the first 80 groups is $90 \%$. The accuracy of the result was validated and could satisfy the requirement when $r$ is 80 . This paper recommends $\lambda_{\text {sum }}$ to be $90 \%$ for these kinds of structures, implying that the computational cost can be reduced by $92 \%$ without sacrificing accuracy.

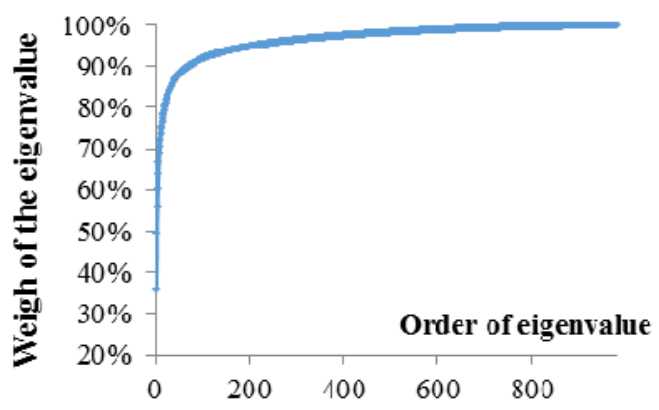

Figure 14. Accumulative Weight Graph of Eigenvalue

\subsection{Change in Wind-induced Response with Frequency Range}

Figure 2 shows that wind load rarely contains high-frequency components. This study explored the effect of high-frequency components of wind load on the response of long-span spatial structures. Figure 15 illustrates the relationship between the mean square deviation of displacement response and frequency of load. The displacement response has mutations at natural frequency of structure, and this phenomenon is most obvious at the first-order natural frequency. The displacement response is extremely small after the first-order natural frequency.

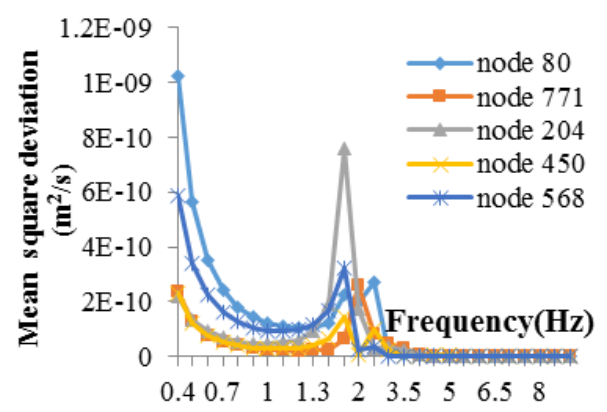

Figure 15. Reflected Power Spectrum Curve of Nodes

Figure 16 shows that the mean square deviation of displacement did not change after the first-order natural frequency. Thus, the high-frequency part of wind load slightly affected the response of long-span spatial structures. The highest frequency of the wind load can be obtained as the first-order natural frequency of long-span spatial structures, which can substantially reduce the computational cost. 

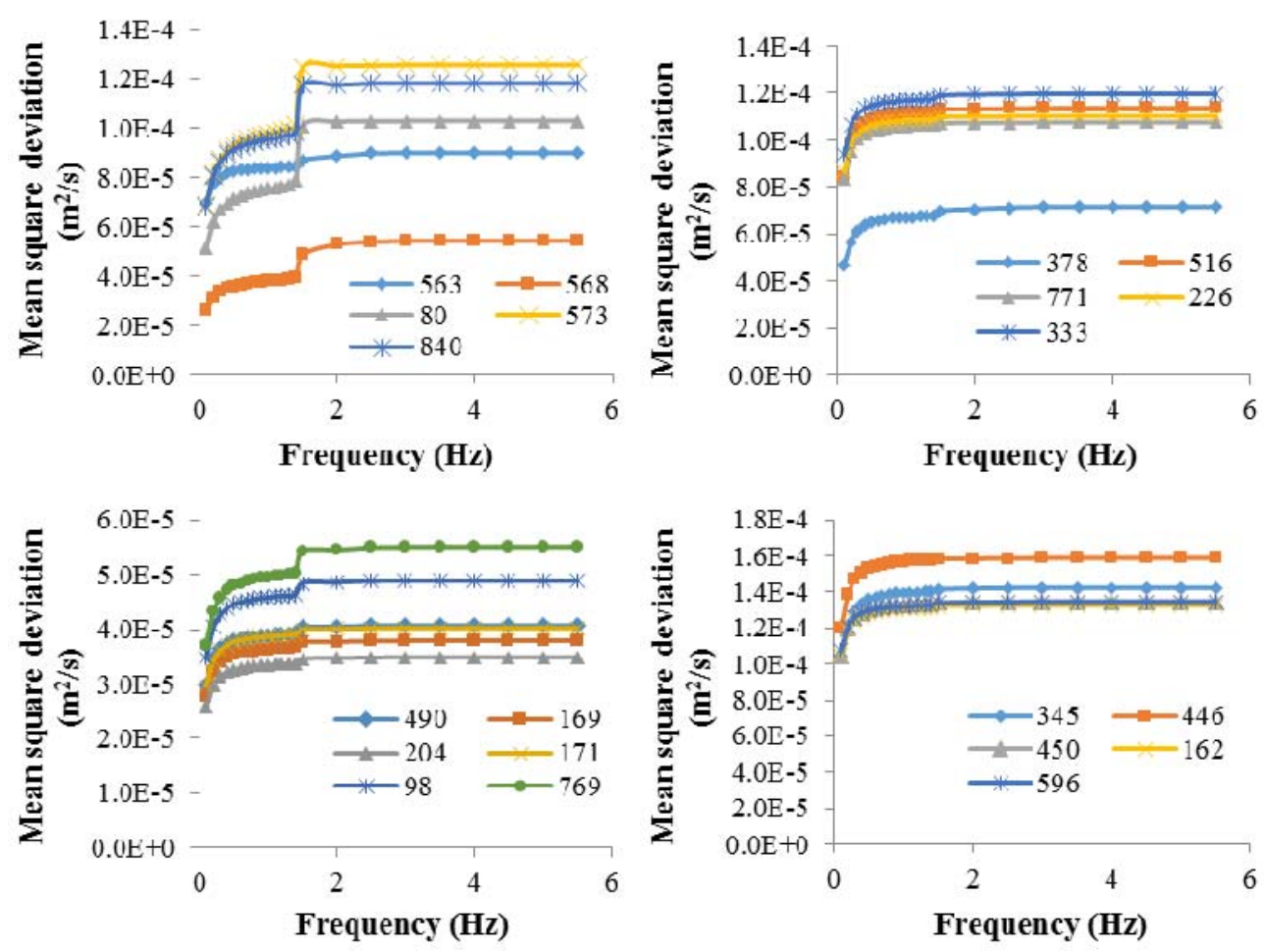

Figures 16. Relationship between Response and Frequency

\subsection{Comparison of Results Derived Using Different Methods}

To reflect the accuracy and efficiency of the POD-pseudo-excitation method, this paper provides the contour of the wind vibration coefficient calculated by different methods as shown in Figure 17. The results obtained by the LDLT-pseudo-excitation and POD-pseudo-excitation methods were consistent. Therefore, they are equivalent in the mathematical sense. From (b) and (c), we conclude that the first 80 order groups of pseudo-excitation can be used to represent the entire random wind field, guaranteeing the accuracy and substantial reduction of computational cost. Comparison of (a), (b), and (d) shows that the results obtained using the time-domain and frequency-domain methods were consistent, and the computational cost was reduced by $92 \%$, establishing the foundations for research on high-efficiency computational technique in the frequency domain for wind-induced analysis of long-span spatial structures.

\section{CONCLUSIONS}

This paper conducted a wind-induced response analysis by using POD-pseudo-excitation method combined with secondary development of ANSYS general purpose software package. Wind vibration coefficient was obtained. Based on an analysis of the results, the following conclusions are presented:

- A new method that can be efficiently used in wind-induced response analysis for long-span spatial structures was proposed. Through the combination of proper decomposition technique and pseudo-excitation method, the computational cost was considerably reduced and the accuracy was validated. This method can be conveniently used by secondary development of mature software package. 
- The first-order natural frequency can be used as the upper limit of wind load frequency for the object to reduce computational cost, and the accuracy can be guaranteed for spatial structures.

- Comparison of the results obtained by the time-domain and frequency-domain methods shows that the results indicated were consistent with each other, and the computational cost can be reduced by $92 \%$ without sacrificing the accuracy.
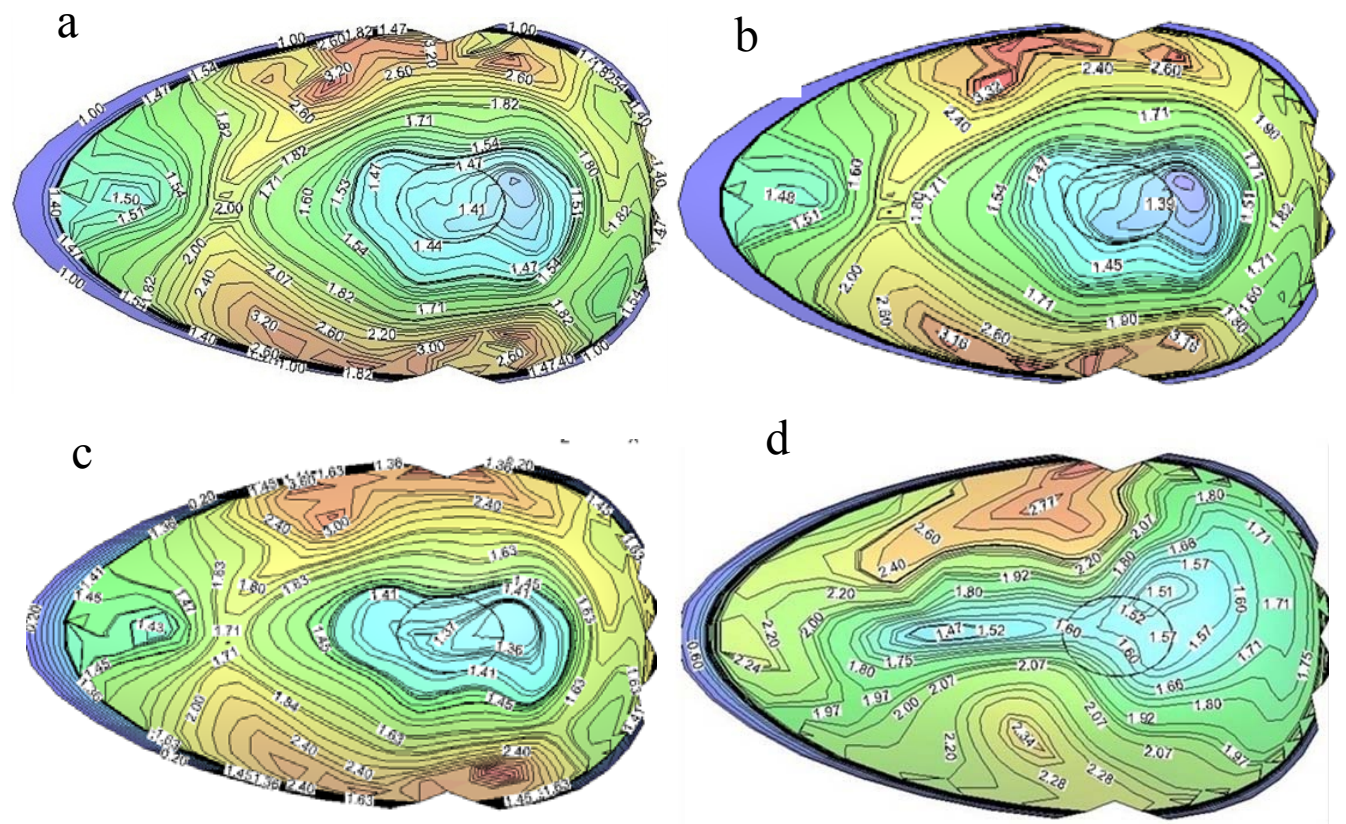

Figures 17. Comparison of Wind Vibration Coefficient. (a) LDLT-pseudo-excitation method, (b) POD-pseudo-excitation method $(r=980)$, (c) POD-pseudo-excitation method $(r=80)$, and (d) dynamic time history analysis.

\section{ACKNOWLEDGEMENT}

The work described in this paper was supported by the scientific and technical project plan of the Ministry of Housing and Urban-Rural Development of China (No. 2015-K2-027) and the Special Funds for Author of Outstanding Doctoral Dissertation (No. 201453)

\section{REFERENCES}

[1] Bian, J.F. and Wei, D.M., "Wind Velocity Time-history Numerical Simulation Theories in Long-span Spatial Structures”, Journal of Jinan University, 2005, Vol. 26, No. 1, pp. 87-90.

[2] Zhang, J.S., Wu, Y. and Shen, S.Z., "Study on Wind-induced Vibration of Single-layer Cylindrical Reticulated Shell Structures”, Industrial Construction, 2006, Vol. 36, No. 10, pp. 69-72.

[3] Samali, B., Yang, J.N. and Yeh, C.T., "Control of Lateral-torsional Motion of Wind-excited Buildings”, Journal of Engineering Mechanics, 1985, Vol. 111, No. 6, pp. 777-796.

[4] Xu, Y.L., Samali, B. and Kwok, K.C.S., "Control of Along-wind Response of Structures by Mass and Liquid Dampers", Journal of Engineering Mechanics, 1992, Vol. 11, No. 81, pp. 20-39.

[5] Zhou, Z., Li, Z.M., Meng, S.P. and Jing, W., "Wind-Induced Vibration Responses of Prestressed Double-layered Spherical Latticed Shells", International Journal of Steel Structures, 2011, Vol. 11, No. 2, pp. 191-202. 
[6] Chen, S.R., Cai C.S., Chang, C.C. and Gu, M., "Modal Coupling Assessment and Approximated Prediction of Coupled Multimode Wind Vibration of Long-span Bridges", Journal of Wind Engineering and Industrial Aerodynamics, 2004, Vol. 92, No.5, pp. 393-412.

[7] Lin, J.H., "A Fast CQC Algorithm of PSD Matrices for Random Seismic Responses", Computers and Structures, 1992, Vol. 44, No.3, pp. 683-687.

[8] Lin, J.H., Sun, D.K., "Application of Pseudo-excitation Method to 3D Buffeting Analysis of the Ting Ma Long-span Suspension Bridge”, Journal of Dalian University of Technology, 1999, Vol. 39, No. 2, pp. 172-179.

[9] Lin, J.H., Zhang, W.S. and Li, J.J., "Structural Response to Arbitrarily Coherent Stationary Random Excitations", Computers \& Structures, 1994, Vol. 50, No. 5, pp. 629-634.

[10] Xu, Y.L, Zhang, W.S., Ko, J.M and Lin, J.H., "Pseudo-excitation Method for Vibration Analysis of Wind-excited Structures", Journal of Wind Engineering and Industrial Aerodynamics, 1999, Vol. 83, No. 1, pp. 443-454.

[11] Chen, B., Yang, Q.S. and Wu, Y., "Wind-Induced Response and Equivalent Static Wind Loads of Long Span Roofs", Advances in Structural Engineering, 2012, Vol. 15, No. 7, pp. 1099-1114.

[12] Yang, Q.S. and Tian, Y.J., “Wind-induced Responses of Beijing National Stadium”,Wind and Structures,2011, Vol.14, No.3, pp.239-252.

[13] Zhou, X.Y. and Gu, M., "an approximation method for computing the dynamic responses and equivalent static wind loads of large-span roof structures", International Journal of Structural Stability and Dynamics, 2010, Vol.10, No.5, pp. 1141-1165.

[14] Bienkiewicz, B., Tamura, Y., Ham, H.J., Ueda, H. and Hibi, K., "Proper Orthogonal Decomposition and Reconstruction of Multi-channel Roof Pressure", Journal of Wind Engineering and Industrial Aerodynamics, 1995, Vol.54, No. 55, pp. 369-381.

[15] Chen, B., Wu, Y. and Shen, S.Z., "The Principle and Application of Ritz-POD Method", Chinese Journal of Computational Mechanics, 2007, Vol. 24, No. 4, pp. 499-504.

[16] Zhang, W.F., Ma, C.H. and Xiao Y., "Some Issues on AR Models for Wind Field Simulation", Chinese Journal of Computational Mechanics, 2009, Vol. 26, No. 1, pp. 124-130.

[17] Yuan, B., Ying, H.Q. and Xu, J.W., "Simulation of Turbulent Wind Velocity Based on Linear Filter Method and MATLAB Program Realization”, Structural Engineers, 2007, Vol. 23, No. 4, pp. 55-61. 